\title{
ON THE DUAL SPACE OF A WEIGHTED BERGMAN SPACE ON THE UNIT BALL OF $\mathrm{C}^{n}$
}

\author{
J.S. CHOA and H.O. KIM \\ Department of Applied Mathematics \\ KAIST \\ P.0. Box 150, Cheongryang \\ Seou1, KOREA
}

(Received January 30, 1987 and in revised form March 24, 1987)

ABSTRACT. The weighted Bergman space $A_{\alpha}^{P}\left(B_{n}\right)(0<p<1)$, of the holomorphic functions on the unit ball $B_{n}$ of $C^{n}$ forms an F-space. We find the dual space of $A_{\alpha}^{P}\left(B_{n}\right)$ by determining its Mackey topology.

KEY WORDS AND PHRASES. Hardy space, Bergman space, Mackey topology. 1980 AMS SUBJECT CLASSIFICATION CODE. 30D55, 32A35.

1. INTRODUCTION.

Let $B_{n}$ be the unit ball of $C^{n}, \nu$ be the normalized Lebesgue measure and $\sigma$ be the rotation invariant positive Borel measure on $S$, the boundary of $B_{n}$, with $\sigma(S)=1$. The weighted Bergman space $A_{\alpha}^{P}\left(B_{n}\right)(0<p<\infty, \alpha \geq-1)$ consists of all functions holomorphic in $B_{n}$ for which

$$
\|f\|_{p, \alpha}^{p}= \begin{cases}\int_{0}^{1} M_{p}^{p}(r ; f)(1-r)^{\alpha} d \sigma(\zeta) 2 n r^{2 n-1} d r<\infty, & \text { if } \alpha>-1, \\ \sup _{0 \leqq r<1} M_{p}^{p}(r ; f)<\infty, & \text { if } \alpha=-1,\end{cases}
$$

where $M_{P}^{P}(r ; f)=\int_{S}|f(r \zeta)|^{P} d \sigma(\zeta)$. Note that the weighted Bergman space $A_{\alpha}^{P}\left(B_{n}\right)$ is, in fact, the Hardy space $H^{P}\left(B_{n}\right)$ if $\alpha=-1$ (See [1]).

The purpose of this paper is to compute the dual space $\left(A_{\alpha}^{p}\left(B_{n}\right)\right)^{*}$ for $0<p \leq 1$ by determining the Mackey topology of $A_{\alpha}^{p}\left(B_{n}\right)$. The corresponding problems for the case $n=1$ are settled by Duren, Romberg and Shields [2], Shapiro [3] and Ahern [4]. Our computations are very similar to those of them.

Throughout this work, $\mathrm{C}_{\alpha, \beta}, \ldots$ denotes a positive constant depending only on $\alpha, \beta, \ldots$ which may vary in the various places, and the notation $a(z) \sim b(z)$ means that the ratio $a(z) / b(z)$ has a positive finite limit as $|z| \rightarrow 1$.

2. SOME PRELIMINARY RESULTS.

LEMMA 2.1. If $\mathrm{f} \in \mathrm{A}_{\alpha}^{\mathrm{p}}\left(\mathrm{B}_{\mathrm{n}}\right)(0<\mathrm{p}<\infty, \alpha \geqq-1)$, then

$$
|f(z)| \leqq c_{n, p, \alpha}\|f\|_{p, \alpha}(1-|z|)^{-\frac{n+\alpha+1}{p}} \text {. }
$$


PROOF. The case $\alpha=-1$ is proved in [1, Thm 7.2.5]. For the proof of the case $\alpha>-1$, it is enough to prove the result for $\frac{1}{2}|z|<1$ since $|f(z)|$ is bounded for $|z| \leqq \frac{1}{2}$. For this range of $\rho$ we have:

$$
\begin{aligned}
\|f\|_{p, \alpha}^{p} & =\int_{0}^{1} \int_{S}|f(r \zeta)|^{p}(1-r)^{\alpha} 2 n r^{2 n-1} d r d \sigma(\zeta) \\
& \geqq C_{n, p} \int_{\rho}^{1} \int_{S}|f(r \zeta)|^{P}(1-r)^{\alpha} d \sigma(\zeta) d r \\
& \geqq C_{n, p} M_{p}^{p}(\rho ; f) \int_{\rho}^{1}(1-r)^{\alpha} d r \\
& =C_{n, p, \alpha} M_{p}^{p}(\rho ; f)(1-\rho)^{\alpha+1} .
\end{aligned}
$$

By the result of the case $\alpha=-1$ and the above result, we get

$$
\begin{aligned}
|f(\rho z)| & \leqq C_{n, p} M_{p}(\rho ; f)(1-|z|)^{-\frac{n}{p}} \\
& \leqq C_{n, p, \alpha}\|f\|_{p, \alpha}(1-\rho)^{-\frac{\alpha+1}{p}}(1-|z|)^{-\frac{n}{p}} .
\end{aligned}
$$

Consequently we have

$$
\begin{aligned}
|f(z)| & =|f(\sqrt{r} \sqrt{r} \zeta)| \quad(z=r \zeta) \\
& \leqq C_{n, p, \alpha}\|f\|_{p, \alpha}(1-\sqrt{r})^{-\frac{\alpha+1}{p}}(1-\sqrt{r})^{-\frac{n}{p}} \\
& \leqq C_{n, p, \alpha}\|f\|_{p, \alpha}(1-r)^{-\frac{n+\alpha+1}{p}} .
\end{aligned}
$$

COROLLARY 2.2. (a) The convergence of $A_{\alpha}^{p}\left(B_{n}\right)$ with its invariant metric

$$
d(f, g)= \begin{cases}\|f-g\|_{p, \alpha}^{p}, & (0<p<1), \\ \|f-g\|_{p, \alpha}, & (p \geq 1)\end{cases}
$$

implies the uniform convergence on any compact subset of $B_{n}$.

(b) $A_{\alpha}^{p}\left(B_{n}\right)$ is an F-space if $0<p<1$ and a Banach space if $p \geq 1$.

PROOF. (a) follows immediately from Lemma 2.1. The proof of (b) is routine and is omitted.

COROLLARY 2.3. $A_{\alpha}^{p}\left(B_{n}\right) \subset A_{\beta}^{q}\left(B_{n}\right)$ if $0<p<q$ and $\frac{n+\alpha+1}{p}=\frac{n+\beta+1}{q}$. In particular,

$$
A_{\alpha}^{P}\left(B_{n}\right) \subset A_{\sigma}^{1}\left(B_{n}\right) \text {, where } \sigma=\frac{n+\alpha+1}{p}-(n+1) .
$$

PROOF. First, we prove the case $\alpha>-1$. We use Lemma 2.1 in the first inequality of the following. 


$$
\begin{aligned}
\|f\|_{q, \beta}^{q}= & \int_{0}^{1} \int_{S}|f(r \zeta)|^{q}(1-r)^{\beta} 2 n r^{2 n-1} d r d \sigma(\zeta) \\
\leqq & \int_{0}^{1} \int_{S}|f(r \zeta)|^{p_{C}} C_{n, p, \alpha, q}\left[(1-r)^{-\frac{n+\alpha+1}{p}}\right]^{q-p}\|f\|_{p, \alpha}^{q-p} \\
& \quad \times(1-r)^{\beta} 2 n r^{2 n-1} d r d \sigma(\zeta) \\
\leqq & c_{n, p, \alpha, q}\|f\|_{p, \alpha}^{q-p} \int_{0}^{1} \int_{S}|f(r \zeta)|^{p}(1-r)^{\alpha} 2 n r^{2 n-1} d r d \sigma(\zeta) \\
\leqq & C_{n, p, \alpha, q}\|f\|_{p, \alpha}^{q} .
\end{aligned}
$$

This completes the proof of the case $\alpha>-1$. The remaining case is essentially a result of Hardy and Littlewood, but we give a proof using Ahern's technique in [5]. Let $f \in H^{p}\left(B_{n}\right)$. By Lemma 2.1, we have

$$
|f(z)| \leqq K_{n, p}(1-|z|)^{-\frac{n}{p}}\|f\|_{p} \text {. }
$$

Set

$$
M f(\zeta)=\sup _{0 \leqq r<1}|f(r \zeta)|
$$

Then we have

$$
\begin{aligned}
& \int_{0}^{1}|f(r \zeta)|(1-r)^{\left(\frac{1}{p}-1\right) n-1} 2 n r^{2 n-1} d r \\
& \leq k_{n, p}\|f\|_{p} \int_{0}^{\lambda}(1-r)^{-n-1} d r+C_{n, p} M f(\zeta) \int_{\lambda}^{1}(1-r)^{\left(\frac{1}{p}-1\right) n-1} d r \\
& \leq K_{n, p}\|f\|_{p} \frac{(1-\lambda)^{-n}}{n}+C_{n, p} M f(\zeta) \frac{(1-\lambda)^{\left(\frac{1}{p}-1\right) n}}{\left(\frac{1}{p}-1\right) n} .
\end{aligned}
$$

If $M f(\zeta) \leq K_{n, p}\|f\|_{p}$, by setting $\lambda=0$ in (2.2), (2.1) is dominated by $C_{n, p}\|f\|_{p}$. If $M f(\zeta) \geq K_{n, p}\|f\|_{p}$, by setting

$$
\lambda=1-\left[\frac{K_{n, p}\|f\|_{p}}{M f(\zeta)}\right]^{\frac{p}{n}}
$$

in (2.2), (2.1) is dominated by

$$
C_{n, p}\|f\|_{p}^{1-p} M f(\zeta)^{p} \text {. }
$$

Hence, for any $\zeta \in \mathrm{s}$,

$$
(2.1) \leqq C_{n, p}\|f\|_{p}+C_{n, p}\|f\|_{p}^{1-p} M f(\zeta)^{p} .
$$

Integrating (2.3) with respect to $\mathrm{d} \sigma(\zeta)$ over $S$ and using the complex maximal theorem [1, Thm. 5.6.5], we obtain 


$$
\begin{aligned}
& \int_{s} \int_{0}^{1}|f(r \zeta)|(1-r)^{\left(\frac{1}{p}-1\right) n-1} 2 n r^{2 n-1} d r d \sigma(\zeta) \\
& \leq C_{n, p}\|f\|_{p}+C_{n, p}\|f\|_{p}^{1-p}\|f\|_{p}^{p} \\
& \leq C_{n, p}\|f\|_{p} .
\end{aligned}
$$

3. THE MACKEY TOPOLOGY OF $A_{\alpha}^{p}\left(B_{n}\right)$.

In this section, we w111 show that the Mackey topology of $A_{\alpha}^{P}\left(B_{n}\right)$ 1s the restriction of the topology of $A_{\sigma}^{1}\left(B_{n}\right)$, where $\sigma=\frac{n+a+1}{p}-(n+1)$.

First we give necessary definitions.

DEFINITION 3.1. The Mackey topology of a non-locally convex topological vector space $(X, \tau)$ is the unique locally convex topology $m$ on $X$ satiofying the following conditions:

(1) If 1s weaker than $\tau$,

(2) the t-closure of the absolutely convex hull of each $\tau$-nelghborhood of the origin contains an m-nelghborhood of the origin (See [6, Thm 1]).

DEFINITION 3.2. For $\beta>-n$ and $z, w \in B_{n}$, we define

$$
K_{\beta}(z, w)=\left(\begin{array}{c}
n+\beta \\
n
\end{array}\right) \frac{\left(1-|w|^{2}\right)^{\beta}}{(1-<z, w>)^{\beta+n+1}}
$$

and

$$
J_{\beta, \sigma}(w)(z)=\left(1-|w|^{2}\right)^{-0} K_{\beta}(z, w) .
$$

The following proposition 1s useful in the sequel:

PROPOSITION 3.3. $[1, p, 120]$ If $\beta>-n_{\text {, then }} K_{\beta}(x, w)$ 1s aproducing kernel for the holomorph1c functions in $\mathrm{L}^{1}\left\{\left(1-|w|^{2}\right)^{\beta} \mathrm{dv}(w)\right\}$. In other worde, if $f$ is holomorphic on $B_{n}$ and integrable with respect to the measure $\left(1-|w|^{2}\right)^{\beta} d v(w)$, then

$$
f(x)=\int_{B_{n}} K_{\beta}(x, w) f(w) d v(w) .
$$

LEMA 3.4. [1, Prop. 1.4.10] For $2 \in B_{n}$ and $c$ real, we define

$$
I_{c}(z)=\int_{s} \frac{d o(\zeta)}{|1-<z, \zeta>|^{n+c}} \cdot
$$

If $c>0$, then $I_{c}(x) \cdot\left(1-|z|^{2}\right)^{-c}$.

LEMMA 3.5. [7, Lamma 6] If $0<r, p<1$ and $\alpha-\beta+1<0$, then

$$
\int_{0}^{1}(1-r)^{\alpha}(1-\rho r)^{\beta} d r \leq C_{\alpha, \beta}(1-p)^{\alpha-\beta+1}
$$

for some positive constant $C_{\alpha, \beta}$.

The next lemma 1s an easy application of the above two lommas.

LEMMA 3.6. Let $0<p<1$ and $f 1 x \beta>\frac{n+a+1}{p}-(n+1) \equiv \sigma$. Then

$$
\sup \left\{\left\|J_{B, \sigma}(w)\right\|_{p, a}^{p}: w \in B_{n}\right\}<\infty .
$$

PROOF. We only prove the case $\alpha>-1$. Let $w \in B_{n}$, and $0<r<1$. 
Then we have, by Lemma 3.4 and 3.5 ,

$$
\begin{aligned}
& \left\|J_{\beta, \sigma}(w)\right\|_{p, \alpha}^{p} \\
= & \int_{0}^{1} \int_{S}\left|J_{\beta, \sigma}(w)(r \zeta)\right|^{p}(1-r)^{\alpha} 2 n r^{2 n-1} d r d \sigma(\zeta) \\
\leqq & C_{n, p, \beta}\left(1-|w|^{2}\right)^{(\beta-\sigma) p} \int_{0}^{1}(1-r)^{\alpha} \int_{S} \frac{d \sigma(\zeta)}{|1-<r \zeta, w>|^{(\beta+n+1) p}} d r \\
\leqq & C_{n, p, \beta}\left(1-|w|^{2}\right)^{(\beta-\sigma) p} \int_{0}^{1}(1-r)^{\alpha}\left(1-r|w|^{2}\right)^{n-(\beta+n+1) p} d r \\
\leqq & C_{n, p, \alpha, \beta}\left(1-|w|^{2}\right)^{(\beta-\sigma) p}\left(1-|w|^{2}\right)^{\alpha+n+1-(\beta+n+1) p} \\
\leqq & C_{n, p, \alpha, \beta} \cdot
\end{aligned}
$$

Thus we have

$$
\sup \left\{\left\|J_{B, \sigma}(w)\right\|_{p, \alpha}^{p}: w \in B_{n}\right\}<\infty .
$$

The proofs of the following theorems are essentially the same as those of [3] (Prop. 4.4 and Prof. 4.5) and are omitted.

THEOREM 3.7. Let $0<\mathrm{p}<1$ and $\beta>\frac{\mathrm{n}+a+1}{\mathrm{p}}-(\mathrm{n}+1) \equiv \sigma$. Then there exists $C_{n, p, \alpha, \beta}<\infty$ such that for each $f \in A_{\sigma}^{1}\left(B_{n}\right)$ there exist a sequence ( $\left.w_{j}\right)$ of the points in $B_{n}$ and a sequence $\left(\lambda_{j}\right)$ of the complex numbers such that

$$
\sum_{j}\left|\lambda_{j}\right| \leqq c_{n, p, \alpha, \beta}\|f\|_{1, \sigma}
$$

and

$$
f=\sum_{j} \lambda_{j} J_{B, \sigma}\left(w_{j}\right),
$$

where the last series converges in $A_{\sigma}^{1}\left(B_{n}\right)$.

THEOREM 3.8. The Mackey topology of $A_{\alpha}^{p}\left(B_{n}\right)$ is the restriction of the topology of $A_{\sigma}^{1}\left(B_{n}\right)$ where $\sigma=\frac{n+\alpha+1}{p}-(n+1)$.

4. THE DUAL SPACE OF $\mathrm{A}_{\alpha}^{\mathrm{p}}\left(\mathrm{B}_{\mathrm{n}}\right)$.

Finally, we will find the dual space of $A_{\alpha}^{P}\left(B_{n}\right)$. For the proof of this main result, the following definition is needed:

DEFINITION 4.1. (Radial fractional derivatives of holomorphic functions in $B_{n}$ ) Let $g(z)=\sum_{k=0}^{\infty} G_{k}(z)$ be the homogeneous expansion of $g$. For any real number $q$, the radial fractional derivative of $g$ of order $q$ is defined by

$$
R^{q} g(z)=\sum_{k=0}^{\infty}(k+1)^{q} G_{k}(z) .
$$

Let

$$
f(z)=\sum_{k=0}^{\infty} F_{k}(z)=\sum_{k=0}^{\infty} \sum_{|\gamma|=k} c(\gamma) z^{\gamma},
$$


and

$$
g(z)=\sum_{\ell=0}^{\infty} G_{\ell}(z)=\sum_{\ell=0|\delta|=\ell}^{\infty} d(\delta) z^{\delta}
$$

be the homogeneous expansions of $f$ and $g$, respectively. We note that for $q>0$, $0 \leqq \rho<1$, we have

$$
\begin{aligned}
& \sum_{k=0|\gamma|=k}^{\infty} c(\gamma) \overline{d(\gamma)} \frac{(n-1) ! k !}{(n-1+k) !} \frac{2 n(k+1)^{q}}{(k+n)^{q}} \rho^{k} \\
= & \frac{2^{q}}{\Gamma(q)} \int_{0}^{1}\left(\log \frac{1}{r}\right)^{q-1} \int_{S} R^{-a} f(r \zeta) \overline{R^{q+a} g(r \rho \zeta) 2 n r^{2 n-1} d r d \sigma(\zeta) .}
\end{aligned}
$$

We can now prove the duality relation. We use the idea of Ahern [4] in the proof of the following.

THEOREM 4.2. Let $0<p<1$ and $\sigma=\frac{n+a+1}{p}-(n+1)$. Then

$$
\left(A_{\alpha}^{p}\left(B_{n}\right)\right)^{*}=\left\{f \in H\left(B_{n}\right): \sup (1-|z|)\left|R^{\sigma+2} f(z)\right|=\|f\|_{\Lambda_{1}}<\infty\right\}
$$

PROOF. By Theorem 3.8, $\left(A_{\alpha}^{\mathrm{p}}\right)^{\star}=\left(\mathrm{A}_{\sigma}^{1}\right)^{\star}$. It suffices to compute $\left(\mathrm{A}_{\sigma}^{1}\right)^{*}$. For simplicity we assume $\sigma=0$. Take $g$ such that

$$
\sup _{z \in B_{n}}(1-|z|)\left|R^{2} g(z)\right|<\infty
$$

and let $f$ be a polynomial. Then by (4.1)

$$
\begin{aligned}
& \sum_{k=0|\gamma|=k}^{\infty} c(\gamma) \overline{d(\gamma)} \frac{(n-1) ! k !}{(n-1+k) !} \frac{2 n(k+1)^{q}}{(k+n)^{q}} \rho^{k} \\
= & 2 \int_{0}^{1} \int_{S} R^{-1} f(r \zeta) \overline{R^{2} g(r \rho \zeta)} 2 n r^{2 n-1} d r d \sigma(\zeta) \\
= & 4 \int_{0}^{1} \int_{S}\left(\log \frac{1}{r}\right) f(r \zeta) \bar{R}^{2} g(r \rho \zeta) 2 n r^{2 n-1} \operatorname{drd} \sigma(\zeta) .
\end{aligned}
$$

Hence

$$
\begin{aligned}
& \left|\lim _{\rho \rightarrow 1}\left(\sum_{k=0}^{\infty} \sum_{\gamma \mid=k} c(\gamma) \overline{d(\gamma)} \frac{(n-1) ! k !}{(n-1+k) !} \frac{2 n(k+1)^{q}}{(k+n)^{q}} \rho^{k}\right)\right| \\
& \leq 4 \int_{0}^{1} \int_{S} \frac{10 g !}{1-r}|f(r \zeta)| \sup _{z \in B_{n}}(1-r)\left|R^{2} g(r \zeta)\right| 2 n r^{2 n-1} \mathrm{drd} \sigma(\zeta) .
\end{aligned}
$$

Since $\log \frac{1}{r} \sim 1-r$ as $r \rightarrow 1,(4.2)$ is dominated by

$$
\mathrm{C}_{\mathrm{k}, \mathrm{n}}\|\mathrm{f}\|_{\mathrm{A}_{0}^{1}}\|\mathrm{~g}\|_{\Lambda_{1}}
$$

Since polynomials are dense in $A_{0}^{1}$, the mapping

$$
\psi(f)=\lim _{\rho \rightarrow 1}\left(\sum_{k=0|\gamma|=k}^{\infty} c(\gamma) \overline{d(\gamma)} \frac{(n-1) ! k !}{(n-1+k) !} \frac{2 n(k+1)^{q}}{(k+n)^{q}} \rho^{k}\right)
$$


extends to be a bounded linear functional on $A_{0}^{1}$. Conversely, let $\psi \in\left(A_{0}^{1}\right)^{*}$. Since $A_{0}^{1} \subset L^{1}\left(2 n r^{2 n-1} d r d \sigma(\zeta)\right)$, by the Hahn-Banach theorem $\psi$ extends to be a bounded linear functional $\psi$ on the space $L^{1}\left(2 n r^{2 n-1} d r d \sigma(\zeta)\right)$. But since $\left(L^{1}\right)^{*}=L^{\infty}$, there exists $G$ in $L^{\infty}\left(2 n r^{2 n-1} \mathrm{drd} \sigma(\zeta)\right)$ such that

$$
\psi(f)=\int_{0}^{1} \int_{S} f(r \zeta) \overline{G(r \zeta)} 2 n r^{2 n-1} d r d \sigma(\zeta)
$$

for each $f$ in $A_{0}^{l}$. Let

$$
H(z)=\int_{0}^{1} \int_{S} \frac{G(w)}{(1-<z, w>)^{n+1}} 2 n \rho^{2 n-1} \operatorname{d} \rho d \sigma(n) \quad(w=\rho \eta)
$$

be the holomorphic projection of $G$. If $f$ is a holomorphic polynomial, then

$$
\begin{aligned}
\psi(f)= & \int_{0}^{1} \int_{S} f(r \zeta) \overline{G(r \zeta)} 2 n r^{2 n-1} d r d \sigma(\zeta) \\
& \int_{0}^{1} \int_{S} f(r \zeta) \overline{H(r \zeta)} 2 n r^{2 n-1} d r d \sigma(\zeta) \\
& \int_{0}^{1} \int_{S} R^{-1} f(r \zeta) \overline{\operatorname{RH}(r \zeta)} 2 n r^{2 n-1} d r d \sigma(\zeta) \\
& \int_{0}^{1} \int_{S} R^{-1} f(r \zeta) \overline{R^{2} g(r \zeta)} 2 n r^{2 n-1} d r d \sigma(\zeta),
\end{aligned}
$$

where $g$ is defined to be $R^{-1} H$. The proof will be complete if we can show that

$$
\sup _{z \in B_{n}}(1-|z|)\left|R^{1} H(z)\right|<\infty .
$$

Since

$$
\frac{\partial H(r \zeta)}{\partial r}=\int_{0}^{1} \int_{S} \frac{(n+1)}{r} \frac{<r \zeta, \rho \eta>G(\rho \eta)}{(1-<r \zeta, \rho \eta>)^{n+2}} 2 n \rho^{2 n-1} d \rho d \sigma(\zeta), \quad(z=r \zeta)
$$

we have

$$
\begin{aligned}
r\left|\frac{\partial H(r \zeta)}{\partial r}\right| & \leqq C_{n}\|G\|_{\infty} \int_{0}^{1} \int_{S} \frac{d \sigma(n)}{|1-<r \zeta, \rho n>|^{n+2} d \rho} \\
& \leqq C_{n}\|G\|_{\infty} \int_{0}^{1} \frac{1}{(1-\rho r)^{2}} d \rho \\
& =C_{n}\|G\|_{\infty} \frac{1}{1-r} .
\end{aligned}
$$

By (4.3) and $R^{1} H(r \zeta)=r \frac{\partial H(r \zeta)}{\partial r}+H(r \zeta)$, we have

$$
\sup _{z \in B_{n}}(1-|z|)\left|R^{1} H(z)\right|<\infty
$$




\section{REFERENCES}

1. RUDIN, W. Function Theory in the Unit Ba11 of $C^{n}$, Springer-Verlag, New York, 1980.

2. DUREN, P.L. ROMBERG, W. and SHIELDS, A.L. Linear Functionals on $\mathrm{H}^{\mathrm{p}}$ space with $0<\mathrm{p}<1$, J. Reine Angew. Math. 38 (1969), 32-60.

3. SHAPIRO, J.H. Some F-spaces of Harmonic Functions for which Orlicz-Pettis Theorem Fails, Proc. London Math. Soc. 50 (1985), 299-313.

4. AHERN, P.R. and JEVTIĆ, M. Duality and Multipliers for Mixed Normed Spaces, Michigan Math. J. 30 (1983), 53-64.

5. KIM, H.0. Derivatives of Blaschke Products, Pacific J. of Math. 114 (1984), 175-190.

6. SHAPIRO, J.H. Mackey Topologles, reproducing Kernels and Diagonal Maps on the Hardy and Bergman Spaces, Duke Math. J. 43 (1976), 187-202.

7. SHIELDS, A.L. and WILLIAMS, D.L. Bounded Projections, Duality, and Multipliers in Space of Analytic Functions, Trans. Amer. Math. Soc. 162 (1971), 287-302. 


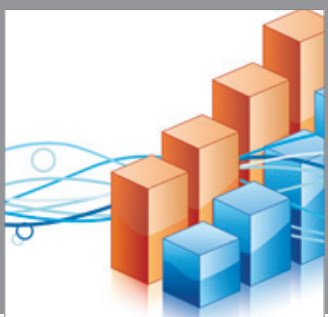

Advances in

Operations Research

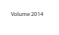

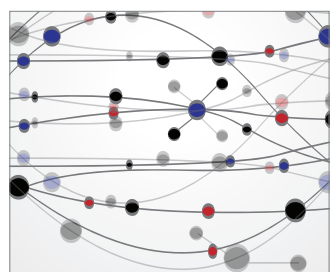

\section{The Scientific} World Journal
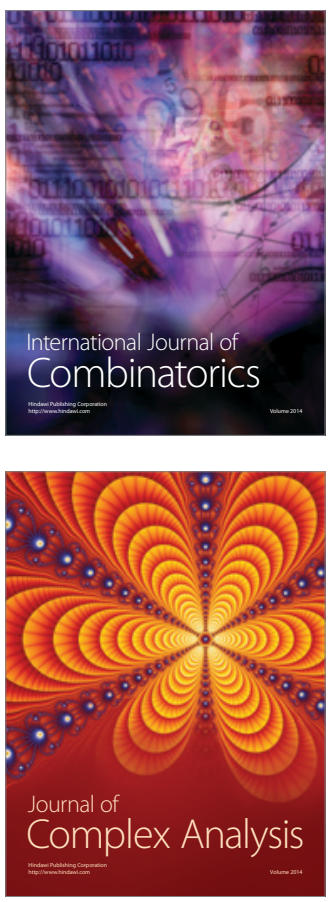

International Journal of

Mathematics and

Mathematical

Sciences
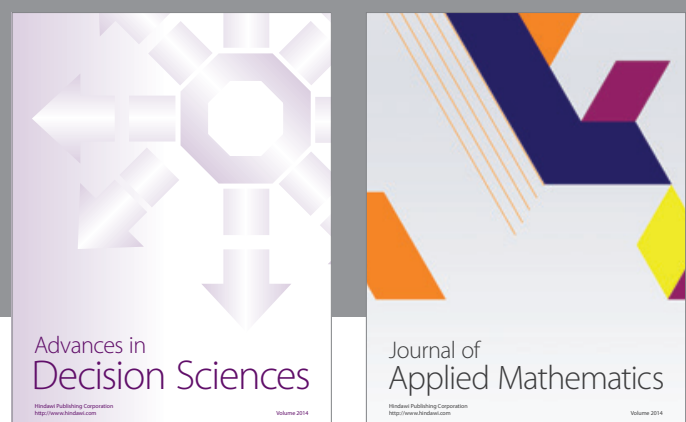

Journal of

Applied Mathematics
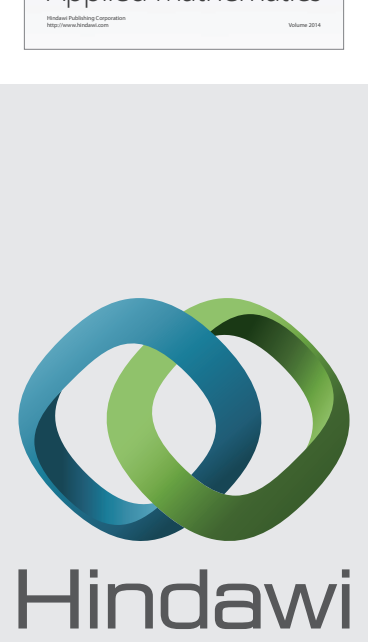

Submit your manuscripts at http://www.hindawi.com
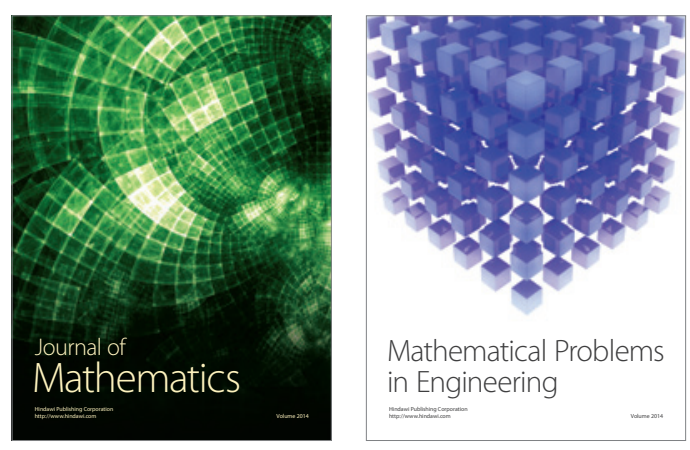

Mathematical Problems in Engineering
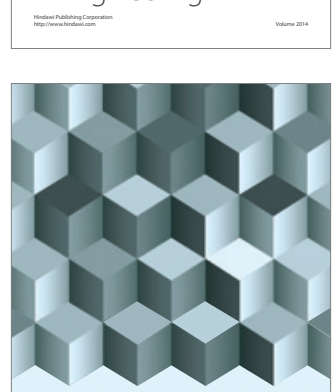

Journal of

Function Spaces
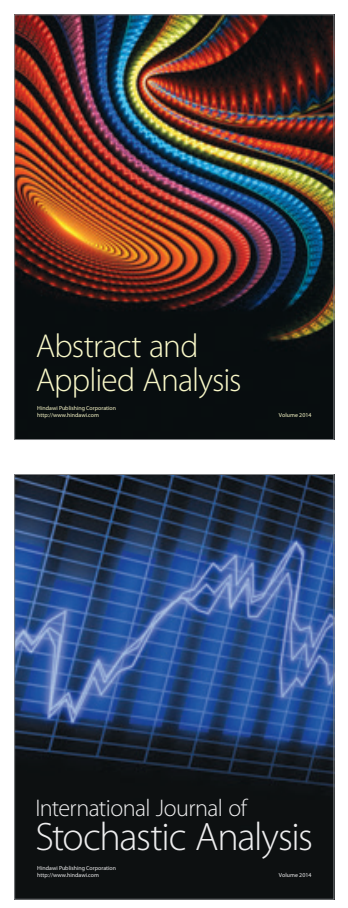

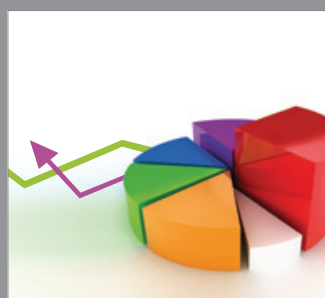

ournal of

Probability and Statistics

Promensencen
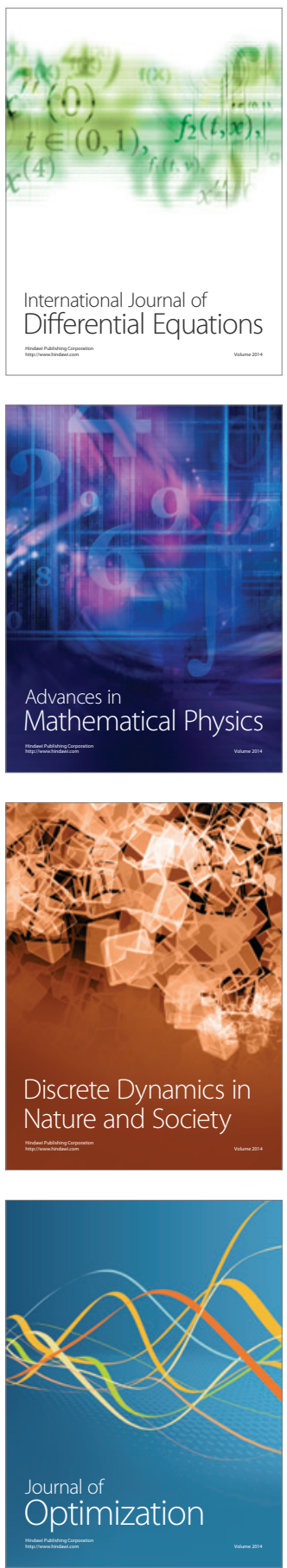